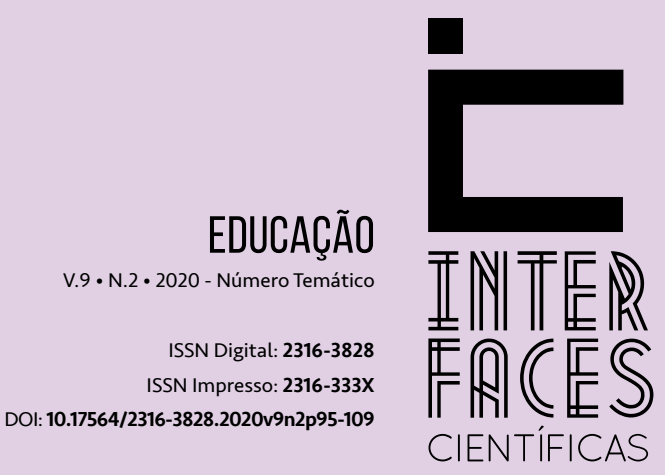

\section{PROJETO PENSAR, CONECTARE FAZER: $O$ USO DAS TECNOLOGIAS DIGITAIS PARA A APRENDIZAGEM DA PROPORCIONALIDADE}

PROJECT TO THINK, CONNECT AND MAKE: THE USE OF DIGITAL TECHNOLOGIES FOR THE LEARNING OF PROPORTIONALITY

PROYECTO PENSAR, CONECTAR Y HACER: EL USO DE LAS TECNOLOGÍAS DIGITALES PARA EL APRENDIZAJE DE LA PROPORCIONALIDAD

Juscileide Braga de Castro ${ }^{1}$ José Aires de Castro Filho²

\section{RESUMO}

Este artigo, considerando que as representações e comunicações possibilitadas pelas tecnologias digitais, trazem para a Educação Matemática uma variedade de formas de expressão e de perspectivas de trabalho em sala de aula, tem como objetivo analisar evidências do potencial das tecnologias utilizadas durante o projeto Pensar, Conectar e Fazer, para a aprendizagem de conceitos relacionados à proporcionalidade. 0 projeto realizado com 12 estudantes do $6^{\circ}$ ano do Ensino Fundamental buscou promover a construção e a reflexão de conceitos Matemáticos a partir do uso de Recursos Educacionais Digitais (vídeos, softwares, simulações, aplicativos) e da produção colaborativa de materiais (vídeos, infográficos, textos argumentativos, dentre outros etc.). Os resultados trazem evidências de que as tecnologias contribuíram para o desenvolvimento da compreensão da proporcionalidade, por meio da visualização e da representação, da construção e produção de conhecimento e da significação.

\section{PALAVRAS-CHAVE}

Projeto. Proporcionalidade. Múltiplas Representações. Tecnologias Digitais. 


\section{ABSTRACT}

Considering that the representations and communications made possible by digital technologies bring to Mathematics Education a variety of forms of expression and work perspectives in the classroom; this article aims to analyze evidence of the potential of the technologies used during the Thinking, Connecting and Doing project; for learning concepts related to proportionality. The project carried out with 12 students from the 6th year of Elementary Education sought to promote the construction and reflection of Mathematical concepts based on the use of Digital Educational Resources (videos, software, simulations, applications) and the collaborative production of materials (videos, , argumentative texts, among others, etc.). The results provide evidence that technologies have contributed to the development of an understanding of proportionality, through visualization and representation; construction and production of knowledge; and meaning.

\section{KEYWORDS}

Project. Proportionality. Multiple Representations. Digital Technologies

\section{RESUMEN}

Considerando que las representaciones y comunicaciones posibilitadas por las tecnologías digitales, traen a la Educación Matemática una variedad de formas de expresión y de perspectivas de trabajo en el aula; este artículo tiene como objetivo analizar evidencias del potencial de las tecnologías utilizadas durante el proyecto Pensar, Conectar y Hacer; para el aprendizaje de conceptos relacionados con la proporcionalidad. El proyecto realizado con 12 estudiantes del $6^{\circ}$ año de la Enseñanza Fundamental buscó promover la construcción y la reflexión de conceptos Matemáticos a partir del uso de Recursos Educativos Digitales (videos, softwares, simulaciones, aplicaciones) y de la producción colaborativa de materiales (videos, infografías, textos argumentativos, entre otros, etc.). Los resultados aportan evidencias de que las tecnologías contribuyeron al desarrollo de la comprensión de la proporcionalidad, por medio de la visualización y la representación; de la construcción y producción de conocimiento; y de la significación.

\section{PALABRAS CLAVE}

Proyecto. Proporcionalidad. Múltiples Representaciones. Tecnologías digitales 


\section{INTRODUÇÃO}

Nos últimos anos, o uso de tecnologias educacionais nas escolas tem se tornado realidade, o que tem suscitado importantes debates sobre suas reais contribuições para a aprendizagem dos alunos. Ainda que a simples disponibilização de aparato tecnológico não seja suficiente para modificar os resultados escolares e alavancar a qualidade da Educação brasileira, a seleção de bons materiais, considerando suas potencialidades para mobilizar habilidades e competências a serem desenvolvidas em cada nível de ensino, assim como, o desenvolvimento de metodologias eficazes, pode contribuir com a aprendizagem dos estudantes e, consequentemente, com a melhoria na qualidade da Educação.

Em relação a qualidade da Educação brasileira é possível verificar, por meio de avaliações de larga escala, como o Sistema de Avaliação da Educação Básica (SAEB), que cerca de 83,30\% dos estudantes brasileiros, cursando o $5^{\circ}$ ano do Ensino Fundamental, não estão no nível considerado adequado em matemática, ou seja, acima de 7, em uma escala que vai de 0 a 10. Isso significa que estes estudantes não são capazes, por exemplo, de resolver problemas que utilizam a multiplicação, envolvendo a noção de proporcionalidade e resolver problemas que envolvem a divisão exata ou a multiplicação de números naturais (BRASIL, 2017a).

A partir deste panorama, é possível inferior que estes estudantes não desenvolveram habilidades esperadas para seu níveel de ensino, como:

(EF05MA08) Resolver e elaborar problemas de multiplicação e divisão com números naturais e com números racionais cuja representação decimal é finita (com multiplicador natural e divisor natural e diferente de zero), utilizando estratégias diversas, como cálculo por estimativa, cálculo mental e algoritmos;

(EF05MA12) Resolver problemas que envolvam variação de proporcionalidade direta entre duas grandezas, para associar a quantidade de um produto ao valor a pagar, alterar as quantidades de ingredientes de receitas, ampliar ou reduzir escala em mapas, entre outros; (EF05MA13) Resolver problemas envolvendo a partilha de uma quantidade em duas partes desiguais, tais como dividir uma quantidade em duas partes, de modo que uma seja o dobro da outra, com compreensão da ideia de razão entre as partes e delas com o todo" (BRASIL, 2017b, [sp]).

Todas estas habilidades são apontadas pela Base Nacional Comum Curricular (BNCC) e estão ligadas a conceitos do campo conceitual Multiplicativo e do campo Algébrico. Segundo a BNCC a habilidade (EF05MA08) faz parte da unidade temática Números, enquanto (EF05MA12) e (EF05MA13) estão indicadas na unidade de Álgebra (BRASIL, 2017b). Dentre os conceitos que são ligados a estas habilidades, pode-se citar: as funções lineares, a fração, a razão, a proporção, a multiplicação e a divisão.

Assim, o desenvolvimento destas habilidades e a compreensão destes conceitos perpassa pelo entendimento de situações multiplicativas. De acordo com Magina, Santos e Merlini (2014), em situ- 
ações multiplicativas é possível verificar o envolvimento entre duas quantidades (de naturezas iguais ou distintas) e uma relação constante entre elas, ou seja, a definição de uma relação fixa, o que requer esquemas mais complexos de pensamento.

0 entendimento desta relação fixa está ligada aos conceitos relacionados com a proporcionalidade, sendo estes, fundamentais para a compreensão da Matemática de uma forma geral, pois, são extremamente pertinentes nas práticas cotidianas da sociedade, por estarem presentes em situações do dia a dia, como na preparação de uma receita, na comparação de preços de produtos com quantidades diferentes, na verificação do montante a ser recebido em relação às horas trabalhadas, na constatação dos quilômetros percorridos em uma viagem, baseado na velocidade e no tempo transcorrido, além de muitas outras situações.

Tyler, Prain e Peterson (2007, p. 317), considerando que os conceitos matemáticos adquirem sentido a partir de um conjunto de situações em que não se é possível isolar um único conceito, explicam que “a aprendizagem de novos conceitos não pode ser separada da maneira de como aprender a representá-los e nem do que significam as representações usadas”. Assim, ao dominar um determinado conceito, espera-se que o estudante seja capaz de mobilizá-lo em diferentes situações e representações.

Entende-se, portanto, que explorar a capacidade de transpor uma representação em outra (tabelas em gráficos, linguagem verbal em diagramas, representação icônica em símbolos, por exemplo), pode trazer um maior significado à compreensão de conceitos. Pesquisas apoiam o uso de múltiplas representações por meio de ambientes computacionais (CASTRO, 2016; BORBA; VILLARREAL, 2005; FERRARA et al., 2006; PIERCE; STACEY, 2001).

Borba e Villarreal (2005) explicam que a utilização de softwares e recursos educacionais no computador podem facilitar as visualizações sem, necessariamente, eliminar a reflexão, possibilitando ainda a formulação de conjecturas, refutações, explicações de conceitos e resultados. Além disso, dinamiza o processo de construção de gráficos e tabelas, por exemplo (CASTRO, 2012; PRAIN; WALDRIP, 2006; PIERCE; STACEY, 2001).

Nesse contexto, o uso de tecnologias para fins educacionais pode trazer vantagens como: a facilidade de visualização e representação de gráficos; as simulações de situações reais; 0 trabalho em contextos investigativos; a produção de conteúdo e informação (CASTRO, 2012). A utilização dos diferentes recursos didáticos disponíveis na escola, como: livro didático, computadores, softwares educacionais, material manipulativo, deve ser planejada e empregada de modo a promover a integração curricular e o desenvolvimento de atividades significativas para os estudantes (CASTRO; CASTRO-FILHO, 2012).

A integração curricular, o uso de múltiplas representações e o envolvimento dos estudantes têm sido propiciados por alguns pesquisadores por meio da aprendizagem baseada em projetos (SILVA; CASTRO; SALES, 2018). Neste tipo de abordagem a aula é centrada no estudante, o que pode promover o desenvolvimento de competências e habilidades diversas.

Diante dessas possibilidades, este artigo tem como objetivo analisar evidências do potencial das tecnologias utilizadas durante projeto Pensar, Conectar e Fazer; para a aprendizagem de conceitos relacionados à proporcionalidade. 
Nas sessões seguintes, apresentar-se-á o referencial teórico e a metodologia adotada nesta investigação. Em seguida, serão discutidos resultados alcançados e apresentadas as conclusões da experiência.

\section{A INTEGRAÇ̃̃O E O USO DE TECNOLOGIAS DIGITAIS}

A dinâmica de crescimento e a evolução dos aparatos tecnológicos que se tem disponível na atualidade têm modificado a forma como as pessoas se comunicam e aprendem (OLIVE, 2011). É possível observar que os recursos digitais estão cada vez mais visuais, interativos e dinâmicos (ARZARELLO; ROBUTTI, 2010; HEGEDUS; MORENO-ARMELLA 2009).

À medida que estas características têm se potencializado em alguns recursos, as possibilidades da utilização de múltiplas representações (textual, numérica, gráfica, simbólica, dentre outras) se ampliam. Atributos como dinamicidade e conexão podem vir a facilitar a realização de trabalhos à distância, por exemplo, ou que um determinado grupo de alunos estejam conectados a fim de trabalhar em conjunto para resolver um mesmo problema. Apesar disso, observa-se que as escolas pouco têm aproveitado estas potencialidades de forma a melhorar o ensino e a aprendizagem da matemática.

As representações e comunicações possibilitadas pela tecnologia digital, trazem para a Educação Matemática uma variedade de formas de expressão e de perspectivas de trabalho em sala de aula.

Hegedus e Moreno-Armella (2009) conseguiram constatar, a partir de mais de 10 anos de investigações realizadas para verificar o impacto da integração de ambientes de softwares dinâmicos, como o Geogebra, por exemplo, que a interseção entre a representação e a comunicação podem trazer mudanças para a aprendizagem, participação e envolvimento dos estudantes. Para isso, eles explicam que é necessário considerar a mobilidade, a exploração de objetos matemáticos em várias representações por meio de diferentes dispositivos e a flexibilidade de capacidade de coleta, manipulação e exibição das diferentes construções (representações).

Outros pesquisadores (DILLENBOURG 1999; STAHL et al., 2006) sugerem que as tecnologias digitais permitem a realização de atividades compartilhadas, oportunizadas por meio de trocas de ideias, discussões, compartilhamento de informação, construção social de conceitos.

Outra questão que tem sido apontada por pesquisas (CASTRO, 2012; 2016; HEGEDUS; MORENO-ARMELLA, 2009) é o envolvimento dos estudantes, ou seja, a participação em atividaes de sala de aula com o uso de tecnologias, como um elemento que pode promover o engajamento em situações de aprendizagem. É importante destacar que a participação e o envolvimento são, sem dúvidas, importantes para a aprendizagem, contudo, o desenvolvimento de ambientes cada vez mais interativos, dinâmicos e sociais requisitam uma análise da natureza dessa aprendizagem, já que possibilitam representações matemáticas por meio de diferentes formas comunicativas.

Mesmo com a existência dessas características, “[...] é preciso ter cuidado para não dar a impressão de que a própria tecnologia faz a diferença no ensino e na aprendizagem. É, naturalmente, não a tecnologia que faz a diferença, mas sim como é usada e por quem" (OLIVE 2011, p. 3).

Apesar de concordar com a afirmação de Olive (2011), é preciso esclarecer que a tecnologia digital tem um papel importante no ensino, caso contrário, não faria sentido utilizá-la. Os diferentes 
usos do potencial das tecnologias digitais têm sido recomendados em documento mais recente do Ministério da Educação (MEC).

Na BNCC é mencionada a necessidade do desenvolvimento de competências ligadas à cultura digital - competência geral 5, que compreende a utilização e a criação de tecnologias digitais, de forma crítica, significativa e ética (BRASIL, 2017b). Além disso, há que se destacar que algumas das habilidades previstas ao longo da Educação Básica requisitam o uso de tecnologias, seja por meio da exploração de softwares de modelagem matemática, na produção de textos com múltiplas linguagens, dentre outros contextos.

A despeito das recomendações, o documento não indica e nem orienta como as escolas e os professors poderão utilizar e integrar as tecnologias em sua prática pedagógica, sendo um grande dificultador para o trabalho docente. Desse modo, entende-se a importância e a necessidade de explorer o potencial das tecnologias, por meio do desenvolvimento de abordagens e/ou metodologias adequadas ao que se quer fazer e, assim, se obterem resultados satisfatórios. A seguir, os procedimentos metodológicos desta investigação serão apresentados.

\section{PROCEDIMENTOS METODOLÓGICOS DA INVESTIGAÇ̃̃O}

Cumpre esclarecer que este trabalho é um recorte da pesquisa de doutorado: Construção do conceito de covariação por estudantes do Ensino Fundamental em ambientes de múltiplas representações com suporte das tecnologias digitais, desenvolvida no âmbito do Programa Observatório da Educação (OBEDUC), por meio do Edital nº 049/2012.

O projeto OBEDUC ao qual este trabalho está vinculado é intitulado: Um estudo sobre o domínio de estruturas multiplicativas no Ensino Fundamental, sendo este executado em rede entre os estados da Bahia, Pernambuco e Ceará, tendo por objetivo geral investigar estratégias que podem ser desenvolvidas e adotadas por professores do Ensino Fundamental para o ensino de conceitos do campo conceitual multiplicativo.

Assim, a pesquisa descrita e analisada neste artigo foi realizada em uma das escolas que participavam do OBEDUC no estado do Ceará. A escolha da escola considerou a disponibilidade da professora de Matemática e da escola, a clientela atendida (anos finais do Ensino Fundamental) e a infraestrutura, já que se trata de uma pesquisa que explora o uso de tecnologias digitais na escola.

A escolha dos 12 estudantes que participarem da pesquisa foi feita pela professora da turma. Neste trabalho os estudantes serão eventualmente caracterizados pela letra “E”, seguida por um número (E01, E02, ... E12) como forma de identificá-los na pesquisa, mas preservar suas identidades.

A intervenção aconteceu no momento das aulas de Matemática, tendo duração de 3 meses, com 18 encontros, divididos em dois momentos: (1) Construção e reflexão acerca de conceitos Matemáticos e (2) Produção colaborativa de vídeo a partir dos conceitos trabalhados nas aulas de Matemática. 0 primeiro momento aconteceu no horário das aulas de matemática em 12 encontros, o segundo, no momento destinado às aulas de disciplina eletiva, com 6 encontros.

Durante o projeto foram utilizados: laptops educacionais; o software Geogebra - para a interpretação e construção de gráficos lineares; o K-word - editor de texto open source, para a produção de 
textos que foram colocados no blog do projeto e para a construção do roteiro dos vídeos; o recurso digital Equilibrando Proporções ${ }^{4}$ - para a exploração de situações de proporcionalidade; o aplicativo Cacoo - usado para a integração de diferentes representações, por meio da construção de infográfi-

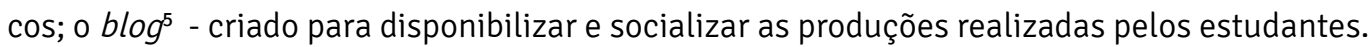

Como forma de continuar as discussões iniciadas em sala de aula e relacionar os conceitos trabalhados com situações reais vivenciados diariamente, foi criado um grupo no aplicativo WhatsApp para ser utilizado por meio do celular e fora da escola.

A análise de dados utilizou-se da técnica de triangulação de dados, já que se empregou diferentes instrumentos (diários de campo, questionários, entrevistas não estruturadas, materiais produzidos pelos estudantes), obtendo-se assim, perspectivas diferentes do mesmo assunto. A seguir, apresentar-se-á a discussão dos resultados.

\section{DISCUSSÃO DOS RESULTADOS}

O Projeto Pensar, Conectar e Fazer teve como objetivo promover a construção e a reflexão de conceitos Matemáticos a partir do uso de Recursos Educacionais Digitais (vídeos, softwares, simulações, aplicativos) e da produção colaborativa de materiais (vídeos, infográficos, textos argumentativos, dentre outros etc.). Os conceitos matemáticos foram desenvolvidos por meio da exploração de ambientes de múltiplas representações integrados a recursos didáticos comumente disponíveis na escola, como o livro didático, por exemplo.

Os resultados dispostos nesta seção apresentam, conforme objetivo do artigo exposto na introdução, evidências do potencial das tecnologias utilizadas durante o projeto Pensar, Conectar e Fazer; para a aprendizagem de conceitos relacionados à proporcionalidade.

De acordo com a análises realizadas, têm-se três categorias: (1) Visualização e representação; (2) Construção e produção de conhecimento; (3) significação, das quais serão detalhadas a seguir.

\section{1 VISUALIZAÇ̃̃O E REPRESENTAÇÃO}

No decorrer do projeto foram propostas diferentes atividades para desenvolver os conceitos relacionados à proporcionalidade, sempre explorando as múltiplas representações. Desta forma, todos os recursos escolhidos contribuíram, de alguma forma, para a visualização e a representação.

A construção dessas representações era feita, especialmente, no Geogebra e no aplicativo online Cacoo (FIGURA 1).

4 http://www.projetos.unijui.edu.br/formacao/_medio/Matematica/EquilibrandoProporcoes/index.html

5 https://pensar-conectar-fazer.blogspot.com/ 
Figura 1 - construção feita com o Cacoo e o Geogebra pelos estudantes E01, E04 e E11

\begin{tabular}{|c|c|c|c|}
\hline GRAMAS & $\mathrm{R} \$$ & GRAMAS & $\mathrm{R} \$$ \\
\hline 30 & 2,49 & 200 & 9,99 \\
\hline 60 & 4,98 & 400 & 19,98 \\
\hline 90 & 9,96 & 600 & 29,97 \\
\hline 200 & 17, & 800 & 39,96 \\
\hline
\end{tabular}

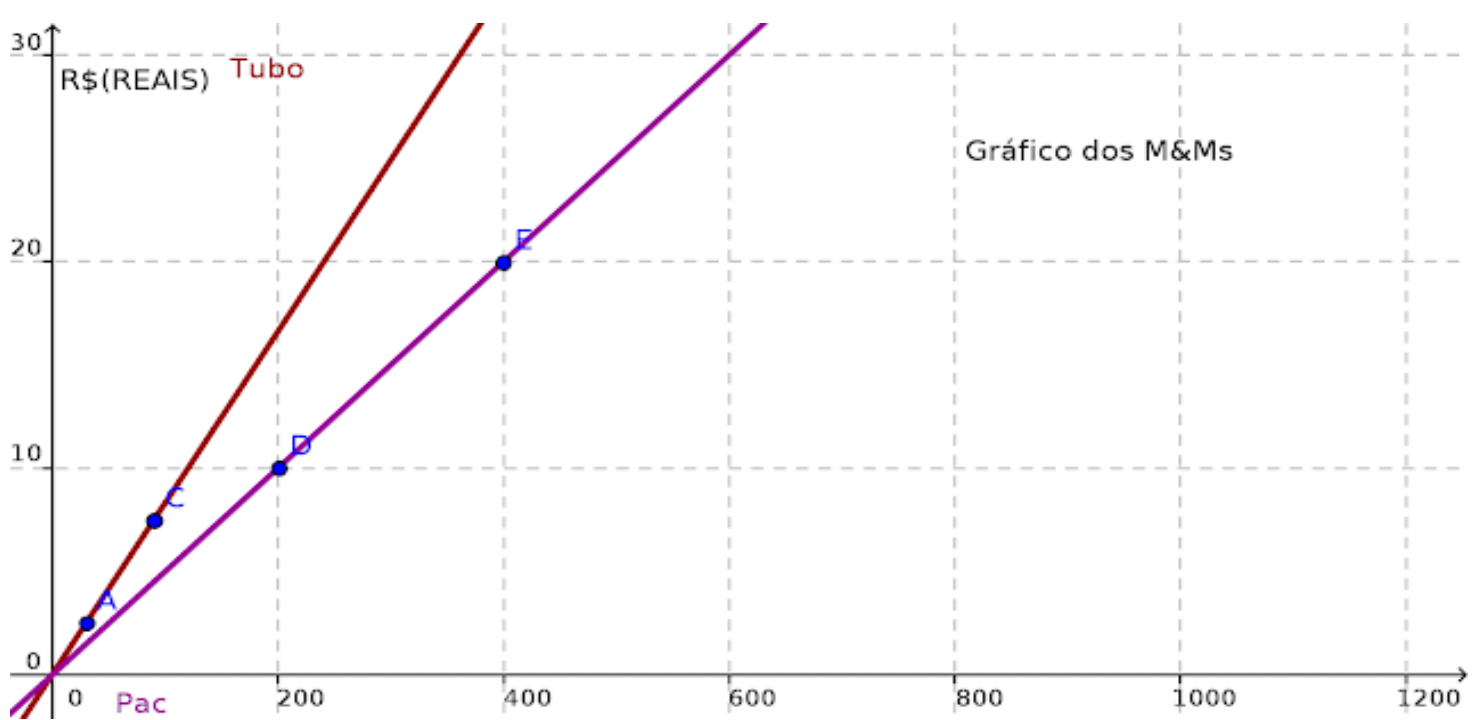

Fonte: Blog do projeto Pensar, conectar e fazer ${ }^{6}$.

A Figura 1 mostra uma atividade produzida por um dos estudantes do projeto com o uso do Caccoe do Geogebra. Nessa situação, os estudantes realizam uma comparação, considerando a relação entre o preço e a quantidade de gramas de um produto. Nesse caso, a representação do gráfico e da tabela são importantes para ajudar na compreensão das relações.

Ressalta-se ainda que a integração destes dois recursos foi importante para explorar seu potencial individual e amenizar algumas das limitações observadas. Verificou-se que o aplicativo Caccoo, por exemplo, não ajudava na precisão e na proporcionalidade necessária na construçao do gráfico,

6 http://pensar-conectar-fazer.blogspot.com/2015/10/o-passeio-doce.html 
mas contribuiu para integrar as diferentes representações (tabelas, figuras, textos, imagens exportadas do Geogebra) por meio da criação de infográficos. Apesar do Geogebra permitir a construção de tabelas, estas não foram bem aceitas pelos estudantes, por acharem o software de difícil manuseio e com limitações estéticas, preferindo fazê-las no Cacoo.

A visualização e as representações puderam, também, ser exploradas por meio de simulações realizadas com o recurso digital Equilibrando Proporções (FIGURA 2).

Figura 2 - Screenshot da tela do Equilibrando proporções

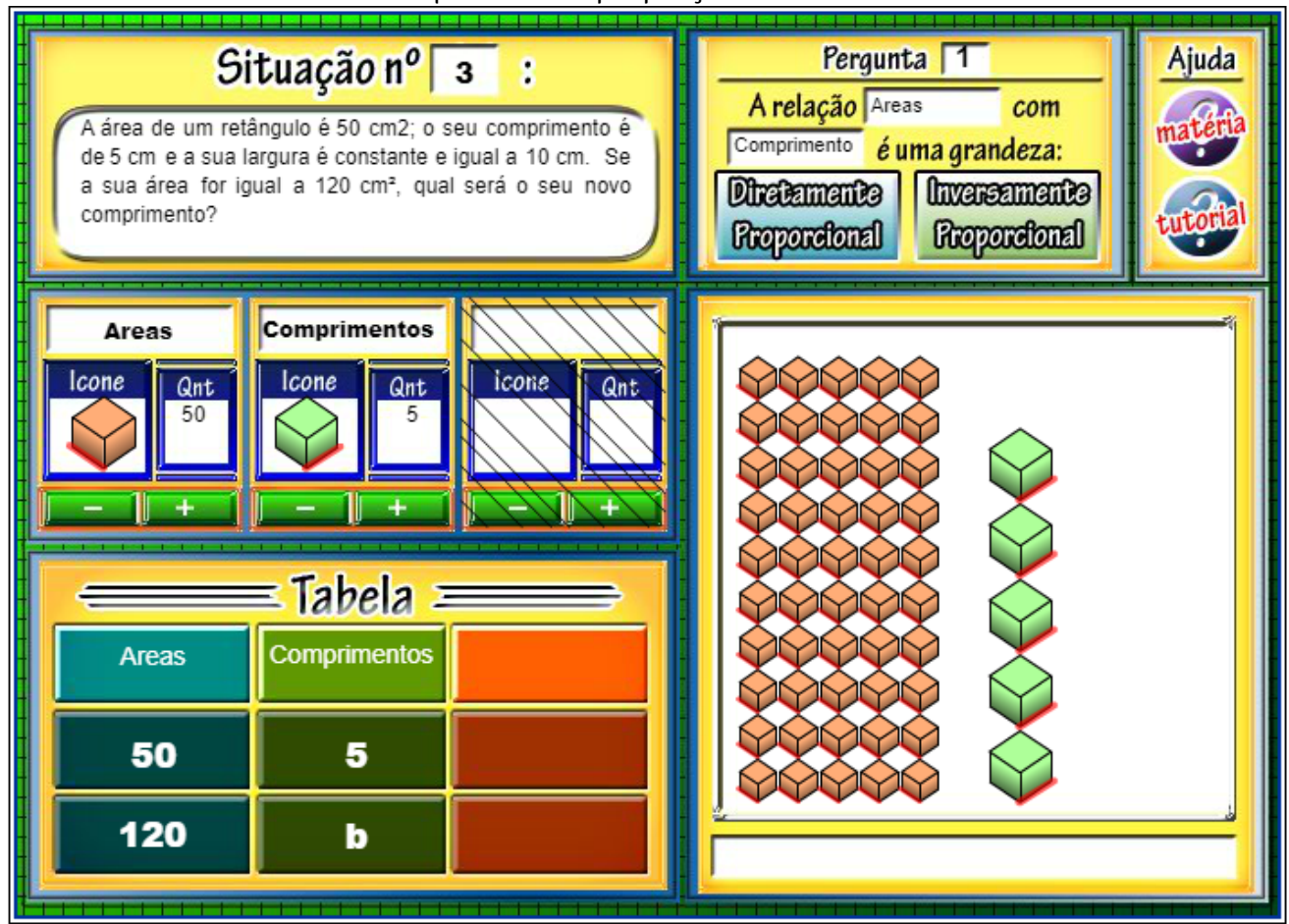

Fonte: http://www.projetos.unijui.edu.br/formacao/_medio/Matematica/EquilibrandoProporcoes/index.html

A partir destas simulações os estudantes puderam utilizar representação icônica (imagens), textual e tabular para inferior as relações existentes nas situações presentes no referido recurso digital. Verificou-se, neste recurso, o potencial de representar os invariantes presentes nas relações entre as grandezas em situações multiplicativas, possibilitando o desenvolvimento da compreensão da proporcionalidade, a partir do estabelecimento das relações entre quantidades distintas e iguais, visualizadas por meio das simulações.

Outro aplicativo que contribuiu com a representação e com a visualização foi o WhatsApp. Embora seu potencial seja reconhecidamente de comunicação, revelou-se um ambiente propicio para a socia- 
lização de estratégias e concepções conceituais. Nesse sentido, os estudantes eram incentivados a fazerem registros diários de suas descobertas em relação às grandezas e postar no grupo do projeto.

As descobertas vinham acompanhadas de registros fotográficos, textos, áudios, tabelas, gráficos, desenhos, emojis, dentre outras formas de representação. Ao postarem essas representações, os estudantes tornaram concreta a concepção de grandeza, possibilitando que todos percebessem a ligação entre a representação e o conceito; demonstrando que " [...] o papel da mídia no processo de visualização vai além do simples ato de mostrar uma imagem” (BORBA; VILLARREAL, 2005).

A seguir serão discutidas as contribuições relacionadas com a construção e com a produção de conhecimento.

\subsection{CONSTRUÇ̃̃O E PRODUÇ̃̃O DE CONHECIMENTO}

A reunião e a interação dos estudantes com diferentes saberes, contribui para consolidação de uma inteligência coletiva, ajudando, assim, a moldar a forma como as pessoas produzem conhecimento.

A partir das análises realizadas, constatou-se que as atividades de interpretação de gráficos, por meio do WhatsApp pode trazer, pelo menos, duas contribuições: a primeira, relacionada a aspectos conceituais da proporcionalidade e a segunda, por propiciar a socialização de diferentes estratégias, por meio da interação social estabelecida no ambiente.

Momentos vivenciados à distância, por meio do WhatsApp superaram, em alguns momentos, as discussões em sala de aula, como: o diálogo ser permeado de exemplos que se tornaram concretos por meio das representações dadas pelos estudantes (imagens, emojis, tabelas, gráficos) e a multimodalidade expressa na participação ativa das crianças, por textos, áudios, vídeos, imagens, que não era tão efetiva em momentos presenciais do projeto.

A construção e a produção de conhecimento também foram verificadas no processo de produção de vídeos, onde os estudantes tiveram que considerar os conceitos trabalhados durante as aulas de matemática. 0 processo de produção de vídeo era realizado em pequenos grupos. Os estudantes (re) definiam os temas de acordo com interesse e curiosidades, assim como, (re)construíam a história que seria contada no vídeo, escolhiam personagens, dentre outros elementos, de modo a contextualizar e dar sentido a situação criada por eles.

Os grupos precisaram ter bem claro os aspectos matemáticos que estariam nas situações criadas, respondendo às seguintes questões: como esse tema pode ser abordado de modo a relacionar com a ideia de multiplicação, divisão e/ou proporcionalidade? Que grandezas serão definidas? Que tipo de relação há entre essas grandezas? Como ficará a representação dessa situação?

Durante a realização do Projeto Pensar, Conectar e Fazer foram produzidos três videos: (1) A grande corrida ${ }^{7}$, (2) Doçura economizada ${ }^{8}$ e (3) Tenha consciência, coma bem! ${ }^{9}$

7 https://www.youtube.com/watch?v=dlLbL0A-08Y\&feature=emb_logo $8 \mathrm{https}: / /$ www.youtube.com/watch?v=Plu4tWqjFDY\&feature=emb_logo 9 https://www.youtube.com/watch?v=RJ3i1qhNKyY\&feature=emb_logo 
Ressalta-se que a criação de vídeo contribuiu não apenas com a produção e construção de conhecimento, mas para a visualização e representação, já que um dos requisitos que os grupos tinham de obedecer era a obrigatoriedade do uso de gráficos. Além disso, também contribuiu para a construção de significados, como será visto a seguir.

\subsection{SIGNIFICACÃ̃}

As representações mentais servem de mediadores na relação do homem com o mundo, as quais estão atreladas ao uso dos instrumentos e de signos como mediadores da atividade humana, impregnados de significado cultural. Como o ser humano desenvolve-se em um ambiente social, essa produção de significados está relacionada à forma do indivíduo interpretar e agir sobre o mundo.

As tecnologias digitais podem ajudar na construção do significado, ao oportunizar a experimentação de ideias, levantamento de hipóteses, formulação de conjecturas, mas também ao possibilitar que o estudante seja confrontado com situações de tomada de decisão. A partir do contexto social, os alunos puderam compreender o significado de algumas relações trabalhadas ao longo do projeto.

0 uso do celular e do aplicativo WhatsApp, permitiu a exploração de situaões reais vivenciadas pelos estudantes fora da escola. Estas situações eram registradas por fotos, vídeos ou anotações, por meio do celular, e socializadas no grupo do WhatsApp ou durante as intervenções em sala de aula. Os estudantes poderam, por exemplo, discutir e constatar a melhor compra a ser feita, considerando produtos de mesma marca, mas com quantidades e preços diferentes.

0 blog do projeto também propiciou significado às construções e às análises feitas pelo grupo, uma vez que, na visão das crianças, serviria para que outras pessoas pudessem entender como fazer uma melhor compra e assim, poder economizar.

Algumas das ideias para os temas dos vídeos criados pelo grupo, surgiram dos infográficos postados no blog. 0 Grupo 2, com vídeo Doçura economizada e o Grupo 3, com Tenha consciência, coma bem! conseguiram definir e desenvolver o roteiro, após vivenciar essas situações de comparações criadas a partir de situações trazidas pelos alunos. 0 vídeo do Grupo 1, A grande corrida, foi originado a partir da curiosidade das crianças em compreender a matemática por trás da caminhada.

Desse modo, ressaltam-se dois aspectos observados em relação à produção de significados: primeiro, o caráter social e segundo, as representações e conceitos matemáticos empregados nos curtas, permitiram que o pensamento coletivo usado na construção desse produto, transitassem do conhecimento imediato e espontâneo, para a formalização e as abstrações matemáticas.

As tecnologias digitais contribuem para a produção de significados, quando servem de instrumento de produção, de transformação de diferentes contextos, pois a significação também está relacionada com o engajamento dos alunos às atividades, o que pode ser constatado pela mudança de postura e comportamento frente ao processo de aprendizagem. Assim, a significação foi verificada em três sentidos distintos: o primeiro, associado ao caráter social que determinada situação está inserida; o segundo, ao significado matemático, relacionado às representações e aos conceitos matemáticos necessários para formalizar as abstrações matemáticas; o terceiro, ligado ao engajamento provocado pelo envolvimento com as atividades e situações abordadas na intervenção. 
Diante dos resultados apresentados constata-se evidências das contribuições das tecnologias digitais. A seguir serão expostas as considerações finais.

\section{CONSIDERAÇÕES FINAIS}

Este artigo analisou evidências do potencial das tecnologias utilizadas durante projeto Pensar, Conectar e Fazer; para a aprendizagem de conceitos relacionados à proporcionalidade. As análises realizadas possibilitam verificar contribuições conceituais proporcionadas pelo uso de tecnologias digitais, relacionadas com: a visualização e a representação; a construção e produção de conhecimento; a significação.

Dentre as atividades que envolviam tecnologias digitais, as discussões promovidas via grupo de WhatsApp, mostraram-se com potencial para a produção de conhecimento, pois a reunião e a interação de pessoas com diferentes saberes favoreceram a consolidação de uma inteligência coletiva. Essa interação contribuiu não apenas para a compreensão conceitual dos estudantes, mas para o desenvolvimento da capacidade de argumentação; de expressão e de representação da matemática; de aprimoração de estratégias em processo de resolução de problemas; competências estas adquiridas ao longo do processo interativo.

Ressalta-se, no referido trabalho, o ganho possibilitado pela diversificação de experiências concretas de forma a propiciar a transposição dos conceitos estudados para contextos reais. É possível concluir que a diversificação de situações e contextos foi ampliada com a exploração da mobilidade (smartphones, laptops, câmera de video) e da comunicação (Whatsapp, blog).

Defende-se a premissa de que as tecnologias digitais contribuem para a produção de significados quando servem de instrumento de produção, de transformação de diferentes contextos, mas também ao possibilitar que o estudante seja confrontado com situações de tomada de decisão e de conflitos de ideias. Ressalta-se, contudo, que todas estas contribuições só são possíveis com mediação.

A pesquisa, ao apontar limitações e/ou contribuições de determinados recursos utilizados, traz indícios de elementos a serem (des)considerados, o que pode fornecer requisitos para a elaboração de outros materiais didáticos, como livros didáticos e recursos digitais, que possam ser utilizados para superarem as dificuldades cognitivas na construção dos conceitos relacionados à proporcionalidade.

\section{REFERÊNCIAS}

ARZARELLO, Ferdinando; ROBUTTI, Ornella. Multimodality in multi-representational environments. ZDM Mathematics Education, v. 42, p. 715-731, 2010.

BORBA, Marcelo C.; VILLARREAL, Monica E. Humans-With-Media and the Reorganization of

Mathematical Thinking: information and communication technologies, modeling, experimentation and visualization'. New York: Springer, 2005. 
BRASIL. Ministério da Educação. SAEB 2017. Brasília: INEP, 2017a.

BRASIL, Ministério da Educação. Base Nacional Comum Curricular. Brasília: MEC/ Secretaria de Educação Fundamental, 2017b.

CASTRO, Juscileide Braga de. A utilização de objetos de aprendizagem para a construção e compreensão de gráficos estatísticos. 2012. Dissertação (Mestrado em Educação) - Programa de Pós-graduação em Educação Brasileira, Universidade Federal do Ceará, Fortaleza-CE, 2012.

CASTRO, Juscileide Braga de. Construção do conceito de covariação por estudantes do ensino fundamental em ambientes de múltiplas representações com suporte das tecnologias digitais. 2016. 275f. Tese (Doutorado) - Programa de Pós-graduação em Educação Brasileira, Universidade Federal do Ceará, Fortaleza-CE, 2016.

CASTRO, Juscileide Braga de; CASTRO-FILHO, José Aires. Projeto um mundo de informações: integração de tecnologias digitais ao currículo escolar. Congresso Brasileiro de Informática na Educação, 1, 2012, Rio de Janeiro. Anais..., Rio de Janeiro: SBC, 2012.

DILLENBOURG, Pierre. What do you mean by collaborative learning? In: DILLENBOURG, P. Collaborative-learning: cognitive and computational approaches. Oxford: Elsevier, 1999. p. 1-19.

FERRARA, Francesca; PRATT, David; ROBUTTI, Ornella. The role and uses of technologies for the teaching of algebra and calculus. In: GUTIÉRREZ, A.; BOERO, P. Handbook of research on the psychology of mathematics education: Past, present and future. Rotterdam: Sense, 2006. p. 237273.

HEGEDUS, Stephen J.; MORENO-ARMELLA, Luis. Intersecting representation and communication infrastructures. ZDM, v. 41, n. 4, p. 399-412, 2009.

MAGINA, Sandra P.; SANTOS, Aparecido; MERLINI, Vera L. O raciocínio de estudantes do Ensino Fundamental na resolução de situações das estruturas multiplicativas. Ciência \& Educação, Bauru, v. 20, n. 2, p. 517-533, 2014.

OLIVE, J. John. Research on technology in mathematics education: theoretical frameworks and practical examples. Seoul: Korea Society of Educational Studies in Mathematics, 2011.

PIERCE, Robyn; STACEY, Kaye. Observations on students responses to learning in a CAS environment. Mathematics Education Reserch Journal, Austrália, v. 13, n. 1, p. 28-46, 2001. 
PRAIN, Vaughan; WALDRIP, Bruce. An exploratory study of teachers and students use ofmulti-modal representations of concepts in primary science. International Journal of Science Education, v. 28, n. 15, p. 1843-1866, 2006.

SILVA, Diego Oliveira da; CASTRO, Juscileide Braga de; SALES, Gilvandenys Leite. Aprendizagem baseada em projetos: contribuições das tecnologias digitais. Tear: Revista de Educação Ciência e Tecnologia, Canoas, v. 7, n. 1, 2018.

STAHL, Gerry; KOSCHMANN, Timothy; SUTHERS, Dan. Computer-supported collaborative learning: an historical perspective. In: SAWYER, R. K. Cambridge handbook of the learning sciences.

Cambridge-UK: Cambridge University, 2006. p. 409-426.

TYTLER, Russell; PRAIN, Vaughan; PETERSON, Suzanne. Representational issues in students learning about evaporation. Research in Science Education, v. 37, p. 313-331, 2007. 
1 Doutora e Mestra em Educação; Licenciada em Matemática; Professora da Universidade Federal do Ceará, vinculada ao Departamento de Teoria e Prática do Ensino, da Faculdade de Educação e no Mestrado Acadêmico em Ensino de Ciências e Matemática do Instituto Federal de Ciência e Tecnologia do Ceará - IFCE; Membro do Grupo de Pesquisa e Produção de Ambientes Interativos e Objetos de Aprendizagem (PROATIVA), desde 2008, onde trabalha em projetos para o desenvolvimento de recursos educacionais digitais, formação de professores e projetos colaborativos em escolas públicas, ligados principalmente à Educação Matemática. E-mail: juscileide@virtual.ufc.br

2 Doutor em Educação Matemática da Universidade do Texas em Austin; Mestre em Psicologia Cognitiva pela Universidade Federal de Pernambuco - UFPE; Professor titular da Universidade Federal do Ceará, leciona no Bacharelado em Sistemas e Mídias Digitais e no Programa de Pós-Graduação em Educação Brasileira da Faculdade de Educação; Líder do Grupo de Pesquisa e Produção de Ambientes Interativos e Objetos de Aprendizagem (PROATIVA), onde trabalha em projetos para o desenvolvimento de recursos educacionais, formação de professores, ligados principalmente à Educação Matemática. E-mail: aires@virtual.ufc.br

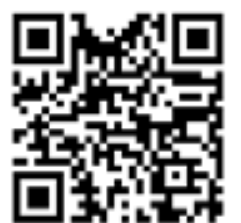

A autenticidade desse artigo pode ser conferida no site https://periodicos. set.edu.br

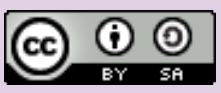

Este artigo é licenciado na modalidade acesso abertosob a Atribuição-Compartilhalgual CC BY-SA

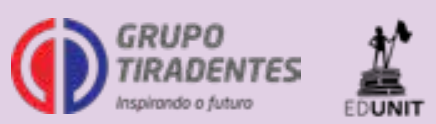

